\title{
THE CIRCUMNUCLEAR REGION IN NGC 4321
}

\author{
J.H. KNAPEN ${ }^{1}$ AND J.E. BECKMAN \\ Instituto de Astrofísica de Canarias, E-38200 La Laguna, \\ Tenerife, Spain \\ 1 Present Address: Université de Montréal, Dépt. de Physique, \\ C.P. 6128, Succ. Centre Ville, Montréal, Québec, H3C-3J7 \\ Canada
}

I. SHLOSMAN AND C.H. HELLER

Department of Physics and Astronomy, University of Kentucky, Lexington, $K Y$ 40506-0055, USA

AND

R.F. PELETIER AND R.S. DE JONG

Kapteyn Astronomical Institute, Postbus 800, NL-9700 AV Groningen, The Netherlands

\begin{abstract}
.
We present results from a recent study of the inner $1 \mathrm{kpc}$ zone of the weakly barred spiral galaxy NGC 4321 (M100), which reveals a circumnuclear starburst, organized in a pair of tightly-wound spiral arms. Combining high-resolution imaging in the optical and near-IR with a detailed dynamical model, we show that the morphology of this region can be explained by a resonance interaction between the disk and the stellar bar and its effects on the gas.
\end{abstract}

NGC 4321 (M100) is a grand-design spiral in the Virgo cluster, which shows a remarkable circumnuclear star-forming structure when seen in $\mathrm{H} \alpha$ (Pogge 1989). The galaxy was classified as Sc by Sandage in the Hubble catalog of galaxies, and as "oval" (SABbc) by de Vaucouleurs et al. (1976), but from near-infrared (NIR) and $\mathrm{H} \alpha$ imaging and from the velocity structure in $\mathrm{HI}_{\mathrm{I}}$ it is clear that NGC 4321 is barred, albeit weakly (Pierce 1986, Knapen et al. 1993). With a very modest $L_{F I R(1-500 \mu m)} / L_{B}=0.42$ (Young 


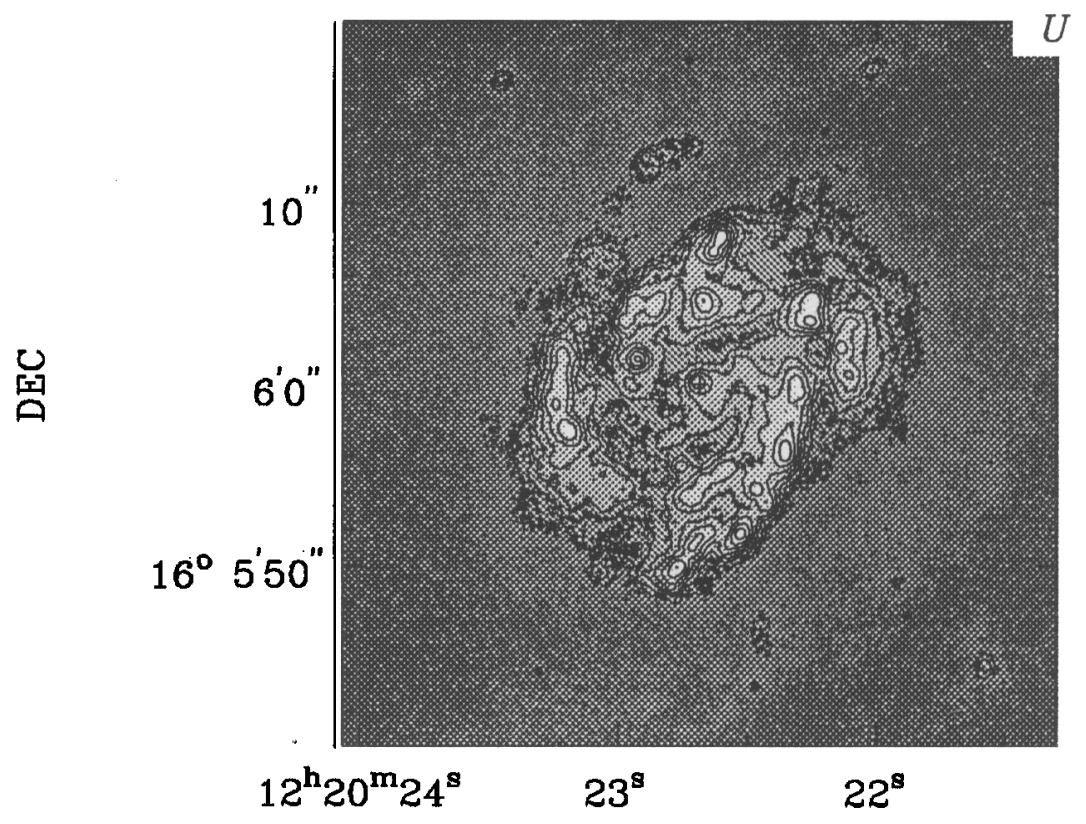

RA

Figure 1. $U$-band image of the inner zone of NGC 4321. The resolution of this image is 0.8 arcsec. Note the spiral arm fragments that are strongly emitting in $U$, indicating massive star formation is taking place there. Dust lanes can be seen coming in from the large-scale bar toward the NW and SE (upper right and lower left in this Figure).

et al. 1989) and an indication of LINER activity in the nucleus (Kennicutt et al. 1989), it is not a "spectacularly active" galaxy .

Non-axisymmetric features in galaxies induce gas inflow towards the central kpc, and the loss of its angular momentum. This enhances the central star formation and can lead to the formation and fueling of a massive black hole there. It is therefore interesting to study the detailed mechanisms leading to the circumnuclear starburst in NGC 4321 and their analogy with Seyfert-type activity in other galaxies.

We have obtained new imaging in the optical and the NIR at subarcsecond resolution using the William Herschel and UK Infrared telescopes to study the inner $1 \mathrm{kpc}$ region of NGC 4321. Our $U$-band image (Fig. 1) shows in detail the spiral arm structure seen by Pogge (1989) in $\mathrm{H} \alpha$. Comparison with Fig. 2, our $K$-band $(2.2 \mu \mathrm{m})$ image, reveals a dramatic change. The star-forming spiral armlets are not seen at all in $K$, and the region inside the armlets is dominated by an elongated structure in the NIR. A 


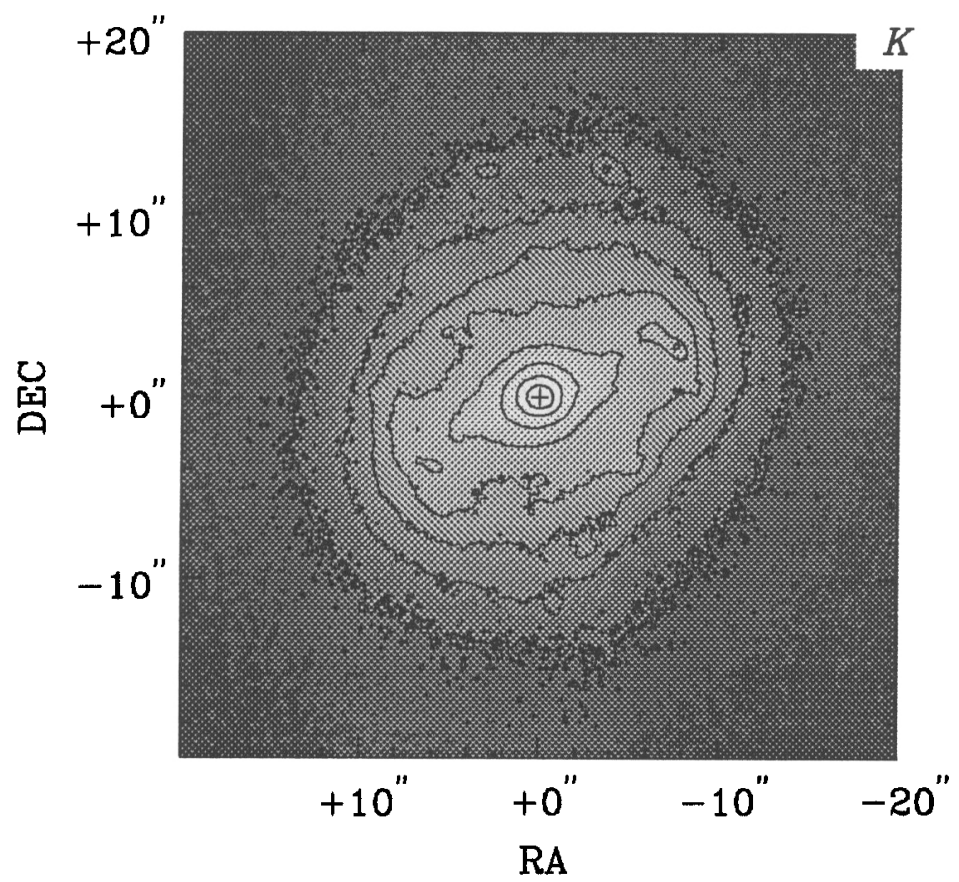

Figure 2. NIR K-band image of the inner region of NGC 4321. Scale and resolution as in Fig. 1.

detailed analysis of the image (Knapen et al. 1995) shows that this structure in fact consists of a small nuclear bulge, an inner bar of some $300 \mathrm{pc}$ radial length ( $1 \mathrm{kpc}$ is 15 arcsec), isophotes gradually skewed in the leading direction with respect to the bar, and finally two regions of intense $K$ emission, symmetrically placed along the direction of the bar $\left(\mathrm{PA}=110^{\circ}\right)$. The inner bar is aligned with the large-scale bar of the galaxy (not shown in the Figures) to within the errors of determination.

From our images in the other optical bands, it is clear that the spiral structure is "washed out" gradually toward redder wavelengths: although images are at the same resolution, star forming peaks are most sharply seen in $U$, and appear smoother in $B, V, R, I$ and eventually $K$, respectively. We interpret this change as stellar evolutionary effects. On one hand, massive stars move away from their birthplaces during their evolution from young and blue to more evolved and redder stars. On the other hand, blue light is emitted more by very massive stars, whereas the contribution from "normal" stars is relatively larger at redder wavelengths. These two effects can erase the spiral structure in the red and NIR and still preserve the twist- 
ing of $K$ isophotes in the leading direction inside the "ring" and in the neighborhood of the inner inner Lindblad resonance (inner ILR). The two symmetric $K$ peaks occur where our model (see below) predicts minima. in the velocity field and are particularly favorable to Jeans instabilities in the gas and to the star formation. A certain amount of gravitational confinement of newly born stars cannot be ruled out there. A fraction of $K$ emission may also be contributed by very hot dust embedded. in this region.

We have performed a 3D self-consistent dynamical modeling of this system using an NGC 4321-like mass distribution in order to study the response of the gas to the underlying gravitational potential, the formation of the nuclear ring-like structure, and the evolution of the system including the effect of star formation. Our numerical method is described in Heller \& Shlosman (1994). After initial transient, the model reaches an approximate steady state in which it accounts for the wealth of morphological features observed. The positions of shocks in the gas corresponds to the positions of the observed dust lanes. Twisting of isophotes in the leading direction as seen in $K$ is related to the gas flow across the inner ILR, and the peaks in $K$ emission occur where outflowing and inflowing gas shocks meet. The star-forming "ring" lies slightly outside the inner ILR whose position is inferred using fully nonlinear analysis.

The combination of observations and modeling shows that the spiral structure observed in the inner $1 \mathrm{kpc}$ zone is a part of a global wave pattern induced by a $4 \mathrm{kpc}$ stellar bar. Gaseous inflow through the bar provides the fueling for enhanced star forming activity between the ILRs and may contribute to the weak LINER nucleus which is embedded in the nonaxisymmetric potential as observed in $K$. A similar mechanism may be at work in active galaxies which harbor the Seyfert nuclei. The differences may include the underlying gravitational potential and the amount of molecular gas finding its way through the ILRs (Shlosman et al. 1989).

\section{References}

de Vaucouleurs, G., de Vaucouleurs, A. \& Corwin, H.G.Jr. 1976, Second Reference Catalogue of Bright Galaxies (RC2), Univ. of Texas Press

Heller, C.H. \& Shlosman, I. 1994, Astrophys. J. 424, 84

Kennicutt, R.C., Keel, W.C. \& Blaha, C.A. 1989, Astron J. 97, 1022

Knapen, J.H., Cepa, J,, Beckman, J.E., del Rio, M.S. \& Pedlar, A. 1993, Astrophys. J. 416, 563

Knapen, J.H., Beckman, J.E., Heller, C.H.,Shlosman, I. \& de Jong, R.S. 1995, in preparation

Pierce, M.J. 1986, Astron J. 92, 285

Pogge, R.W. 1989, Astrophys. J. Suppl. Ser. 71, 433

Shlosman, I., Frank, J. \& Begelman, M.C. 1989, Nature 338, 45

Young, J.S., Xie, S., Kenney, J.D.P. \& Rice, W.L., 1989, Astrophys. J. Suppl. Ser. 70, 699 